\section{LEVEL: A FORTRAN IV program for correlational analysis of group-individual data structures}

\author{
DAVID A. KENNY \\ University of Connecticut, Storrs, Connecticut \\ and \\ JAMES W. STIGLER \\ University of Chicago, Chicago, Mlinois
}

Many multivariate data sets in the behavioral sciences consist of data from individuals nested within groups. Such data can be found in many different fields of research. For example, in educational research, students are often studied within classrooms. In social psychology, individuals often are observed within small groups that are either naturally occurring (e.g., roommates, juries, teams, work groups) or experimentally constituted (e.g., group problem solving).

If the focus is on individual-level processes, researchers often assume independence of observations within groups, and examine correlations that use the individual as the unit of analysis. If group-level processes are the focus, individual observations are aggregated at the group level, and group-level correlations are assumed to reflect group processes.

Many times, however, both levels of analysis are of interest, and it is desirable to consider simultaneously both the individual- and the group-level processes that contribute to the observed correlations. Two factors have discouraged researchers from such simultaneous analyses: First, there has been no convenient computer program that analyzes such data at both levels of analysis in a single run. Second, statistical procedures for partitioning variance into individual and group components have been slow in developing.

A statistical procedure that allows researchers to compute and test variances and correlations at both the group and individual levels for a hierarchically nested design has been described by Kenny and La Voie (in press).

David A. Kenny's mailing address is: Department of Psychology U-20, University of Connecticut, Storrs, Connecticut 06268. James W. Stigler's mailing address is: Department of Psychology, University of Chicago, Chicago, Illinois 60637.
The LEVEL program implements this procedure for partitioning variance into group and individual effects, producing separate covariance and correlation matrices for "persons within groups" and "groups controlling for persons." The program also computes the more traditional, and straightforward, matrices for "persons ignoring groups" and "groups ignoring persons." Missing data and unequal group sizes are allowed. Additional features of the program are described below.

Input. Input to LEVEL is accomplished with five sets of cards (two of which are optional) preceding the data. The first card is a title card. The problem card specifies the number of persons per group (up to 99), the number of groups (up to 99), the number of variables (up to 20), the missing data code, the number of regression equations to be computed (optional; up to 9), and the designation of fixed and random variables. If groups are of unequal size, an optional group-sizes card lists the $\mathrm{N}$ for each group. If the user desires regression equations to be computed, the criterion and predictor variables for each equation are input on an additional card. An inputformat card describes the format in which the data are to be read. Data are input casewise, with cases together by group.

Output. The output is divided into four sections, as described above: persons ignoring groups, groups ignoring persons, persons within groups, and groups controlling for persons. Within each section, the output is arranged as follows: the covariance matrix; the correlation matrix (with $t$ tests above the diagonal); regression coefficients (slope and beta) for each analysis requested, $t$ tests of their significance, and multiple $R$ squared.

Availability. A listing of the program and a user's guide may be obtained free of charge by writing to: David A. Kenny, Department of Psychology U-20, University of Connecticut, Storrs, Connecticut 06268. The program has been run successfully on IBM computers at three different universities. Although it has not been run on other machines, the expectation is that LEVEL should be compatible with minor changes, since no special functions or subroutines are called.

\section{REFERENCE}

Kenny, D. A., \& LA Vore, L. Separating individual and group effects. Journal of Personality and Social Psychology, in press.

(Revision accepted for publication October 17, 1983.) 